\title{
A violência contra mulheres, crianças e adolescentes em tempos de pandemia pela COVID-19: panorama, motivações e formas de enfrentamento
}

\author{
Violence against women, children, and adolescents \\ during the COVID-19 pandemic: overview, \\ contributing factors, and mitigating measures
}

\section{Violencia contra mujeres, niños y adolescentes en tiempos de la pandemia de COVID-19: panorama, motivaciones y formas de afrontamiento}

Emanuele Souza Marques 1

Claudia Leite de Moraes 1,2

Maria Helena Hasselmann 3

Suely Ferreira Deslandes 4

Michael Eduardo Reichenheim 1

doi: 10.1590/0102-311X00074420
A pandemia do novo coronavírus (SARS-CoV-2), causador da doença COVID-19, tem alterado a rotina de grande parte das pessoas. Desde a confirmação do 1o caso da COVID-19 na cidade de Wuhan, China 1 , em dezembro de 2019, até às 17:30 horas do dia 8 de abril de 2020 já haviam 1.500 .830 casos confirmados e 87.706 óbitos ao redor do mundo ${ }^{2}$. Nessa mesma data, o Brasil contabilizava 15.927 casos confirmados e 800 mortos pelo novo coronavírus 3 .

Com o avanço da transmissão da doença nos diversos países e a ocorrência de transmissão comunitária, medidas de contenção social têm sido propostas em diversos países, incluindo o Brasil. Dentre as medidas recomendadas pela Organização Mundial da Saúde (OMS) para o combate à pandemia, destaca-se o isolamento dos casos suspeitos e o distanciamento social, estratégias fundamentais para conter o aumento exponencial dos casos da doença e a sobrecarga no serviço de saúde 4,5. Entretanto, tais recomendações têm severas repercussões negativas para a atividade econômica em todos os seus níveis e para a vida em sociedade. O momento para torná-las imperativas tem sido alvo de discussões e tensões entre pesquisadores, setor produtivo e governantes 6,7. Nesse cenário de disputas entre as medidas a serem adotadas e com uma tímida política pública de apoio financeiro para as populações mais pobres 8 , grande parcela desta população segue sua rotina de trabalho em busca de sustento, sem poder se beneficiar das medidas protetivas do distanciamento social.

Nesse debate, uma questão que vem sendo pouco discutida por pesquisadores, gestores e demais membros dos comitês de crise para o enfrentamento da pandemia são as repercussões do distanciamento social no relacionamento interpessoal, especialmente entre parceiros íntimos e entre pais e filhos. Com base em situações de distanciamento social anteriores e no aumento súbito do registro de casos de violência no contexto de pandemia, organizações internacionais $9,10,11,12,13$, pesquisadores 14,15,16 e mídia leiga 17,18,19,20,21 estão preocupados com os indícios de aumento da violência doméstica, sendo o lar, muitas vezes, um lugar de medo e abuso.

Ao se fazer uma breve revisão sobre o assunto nas mídias sociais e internet, percebe-se que o aumento da violência contra a mulher e contra a criança e ao adolescente durante o período de distanciamento social tem sido observado em diferentes países, tais como China, Reino Unido, Estados Unidos, França e Brasil 22,23,24,25,26,27. Aqui, segundo dados do Ligue 180 disponibilizados pelo Ministério da Mulher, da Família e dos Direitos Humanos houve um aumento de cerca de 17\% no número de ligações com denúncias de violência contra a mulher durante o mês de março, período inicial da
1 Instituto de Medicina Social, Universidade do Estado do Rio de Janeiro, Rio de Janeiro, Brasil. 2 Faculdade de Medicina Universidade Estácio de Sá, Rio de Janeiro, Brasil. 3 Instituto de Nutrição, Universidade do Estado do Rio de Janeiro, Rio de Janeiro, Brasil.

4 Instituto Nacional de Saúde da Mulher, da Criança e do Adolescente Fernandes Figueira, Fundação Oswaldo Cruz, Rio de Janeiro, Brasil.

\section{Correspondência}

E. S. Marques

Instituto de Medicina Social, Universidade do Estado do Rio de Janeiro.

Rua São Francisco Xavier 524, 7o andar, bloco D, Rio de Janeiro, RJ 20550-013, Brasil.

emanuelesm.ims@gmail.com 
recomendação do distanciamento social no país ${ }^{28}$. No Rio de Janeiro, dados do plantão do Ministério Público Estadual revelam um aumento de 50\% nos casos de violência doméstica já no primeiro final de semana após os decretos estaduais que propuseram o distanciamento social, sendo a maior parte das denúncias envolvendo violência contra a mulher 22 . Do mesmo modo, no Paraná, houve um aumento de $15 \%$ nos registros de violência doméstica atendidos pela Polícia Militar no primeiro fim de semana de distanciamento social 29. Situações semelhantes são reportadas no Ceará, Pernambuco e São Paulo 28,30,31.

Instituições que compõem a rede de proteção a mulheres, crianças e adolescentes no Brasil também denunciam o aumento do número de casos e chamam a atenção para a possibilidade de menor visibilidade das situações em função da recomendação de se permanecer em casa, além do fechamento ou redução da jornada de trabalho dos serviços de proteção, tais como a delegacia de mulheres, conselhos tutelares etc. A situação torna-se ainda mais relevante porque em cenários de violência doméstica contra a mulher, na maior parte das vezes, também há violência contra crianças e adolescentes 32,33,34,35,36. Como apontado adiante, a maior incidência ou o acirramento das situações de violência de foro íntimo tem motivações em comum, mas também especificidades.

\section{Fatores que ampliam a vulnerabilidade de mulheres à violência doméstica}

Resgatando-se o modelo ecológico proposto pela OMS para sintetizar as principais dimensões individuais, relacionais, comunitárias e sociais que atuam de forma sinérgica na ocorrência das violências ${ }^{37}$, percebe-se que a crise sanitária, econômica e social trazida pela pandemia COVID-19 e suas necessárias medidas de enfrentamento podem aumentar, sobremaneira, o risco de violência contra a mulher. Para muitas mulheres, as medidas emergenciais necessárias para lutar contra a COVID-19 aumentam o trabalho doméstico e o cuidado com crianças, idosos e familiares doentes. Restrições de movimento, limitações financeiras e insegurança generalizada também encorajam os abusadores, dando-lhes poder e controle adicionais 11 .

A pandemia também traz repercussão no nível comunitário do modelo ecológico, na medida em que diminui a coesão social e o acesso aos serviços públicos e instituições que compõem a rede social dos indivíduos. A busca por ajuda, proteção e alternativas está prejudicada devido à interrupção ou diminuição das atividades em igrejas, creches, escolas e serviços de proteção social, bem como pelo deslocamento das prioridades dos serviços de saúde para as ações voltadas à assistência aos pacientes com sintomas respiratórios e casos suspeitos e confirmados de COVID-19. Esses fatores contribuem de modo a favorecer a manutenção e o agravamento das situações de violência já instaladas.

No âmbito relacional, o maior tempo de convivência com o agressor é crucial. Ademais, ao se reduzir o contato social da vítima com amigos e familiares, reduzem-se as possibilidades de a mulher criar e/ou fortalecer uma rede social de apoio, buscar ajuda e sair da situação de violência. A convivência ao longo de todo o dia, especialmente entre famílias de baixa renda vivendo em domicílios de poucos cômodos e grande aglomeração, reduzem a possibilidade de denúncia com segurança, desencorajando a mulher a tomar esta decisão.

$\mathrm{Na}$ dimensão individual, podem ser estopins para o agravamento da violência: o aumento do nível de estresse do agressor gerado pelo medo de adoecer, a incerteza sobre o futuro, a impossibilidade de convívio social, a iminência de redução de renda - especialmente nas classes menos favorecidas, em que há grande parcela que sobrevive às custas do trabalho informal -, além do consumo de bebidas alcoólicas ou outras substâncias psicoativas. A sobrecarga feminina com o trabalho doméstico e o cuidado com os filhos, idosos e doentes também pode reduzir sua capacidade de evitar o conflito com o agressor, além de torná-la mais vulnerável à violência psicológica e à coerção sexual. O medo da violência também atingir seus filhos, restritos ao domicílio, é mais um fator paralisante que dificulta a busca de ajuda. Por fim, a dependência financeira com relação ao companheiro em função da estagnação econômica e da impossibilidade do trabalho informal em função do período de quarentena é outro aspecto que reduz a possibilidade de rompimento da situação. 


\section{Fatores envolvidos com a violência entre pais e filhos}

Segundo a Organização das Nações Unidas para a Educação, a Ciência e a Cultura (UNESCO), cerca de 1,5 bilhão de crianças e adolescentes em todo o mundo está fora da escola devido ao fechamento das instituições de ensino como iniciativa para a contenção de casos da COVID-19 14,38,39. No Brasil também houve a interrupção das atividades nas creches, escolas e universidades públicas e privadas. Como já comentado, em muitas regiões no país, o fechamento do comércio (exceção para os serviços considerados essenciais), empresas etc., estimulou a realização do trabalho remoto (teletrabalho) para a maior parte dos trabalhadores. Com isso, a dinâmica das famílias com crianças e adolescentes tem exigido um esforço maior dos pais, responsáveis e/ou cuidadores que necessitam conciliar o trabalho remoto, o trabalho doméstico e o cuidado com os filhos.

Reforçando alguns aspectos pontuados na publicação da The Alliance for Child Protection in Humanitarian Action 40 e na declaração de líderes de organizações comprometidas com o combate à violência contra a criança e adolescente 39 , bem como nas questões abordadas anteriormente ao se discutir a violência contra a mulher, acrescentamos outras repercussões que a COVID-19 e suas estratégias de enfrentamento podem trazer para o aumento do risco de violência contra crianças e adolescentes.

No nível social, destacam-se a erosão de suporte social (especialmente o escolar) e questões estruturais relativas à desigualdade de gênero. No nível comunitário, a competição pelos poucos recursos (principalmente na área da saúde), funcionamento parcial de muitos serviços de defesa dos direitos de crianças e adolescentes, bem como a redução das redes sociais podem aumentar o risco de violência. No nível relacional, destaca-se a sobrecarga de trabalho, o estresse dos pais devido às múltiplas tarefas e ao momento que estamos vivendo. As crianças e adolescentes também podem ficar mais irritadiças pelas restrições de mobilidade e pela falta dos colegas, acarretando comportamentos agressivos ou de desobediência. Ademais, o aumento do tempo de convivência, bem como o aumento das tensões nas relações interpessoais, são fatores que podem tornar mais frequentes os episódios de violência contra criança e adolescente neste período. No nível individual, identifica-se a importância de doenças mentais preexistentes e sua possibilidade de agravamento, o que pode diminuir a capacidade de lidar com conflitos e reduzir a supervisão parental.

A situação como um todo e especialmente de distanciamento social pode gerar sentimentos de medo na sociedade. Nas crianças, esses podem ser ainda mais importantes, particularmente quando elas não entendem o que está acontecendo. Ademais, há riscos de perda ou separação dos pais devido ao isolamento/quarentena, o que pode tornar a criança desassistida por um adulto ou, dependendo da idade da criança, o chefe de família 40. Ressalta-se que famílias em situação socioeconômica desfavorável ou residentes em lugares onde há aglomeração são particularmente mais vulneráveis à situação.

\section{Considerações finais}

Diante do exposto, gostaríamos de enfatizar a gravidade da COVID-19 no Brasil e no mundo, e a necessidade de não pouparmos esforços para reduzir a velocidade da transmissão do vírus no nível populacional e reduzir a incidência da doença, especialmente de casos graves. Até o momento, o distanciamento social faz parte do conjunto de medidas necessárias para o alcance desses objetivos. Nessa direção, é mister seguir as recomendações da OMS e do Ministério da Saúde no sentido de fazermos o distanciamento social e a quarentena domiciliar. Entretanto, com base nas evidências e argumentos descritos ao longo deste artigo, reforçamos a necessidade de ações de enfrentamento das violências contra mulheres, crianças e adolescentes.

Diante da percepção de aumento da ocorrência de violências durante o período da pandemia, algumas instituições e organizações sociais têm desenvolvido materiais para a prevenção das violências durante este período de distanciamento social. Dentre as iniciativas existentes, destacam-se os materiais produzidos pela OMS, Fundo das Nações Unidas para a Infância (UNICEF), Centro de Controle e Prevenção de Doenças (Estados Unidos), End Violence Against Children, Internet of Good Things, Parenting for Lifelong Health, Agência para o Desenvolvimento Internacional (Estados Unidos) e End Violence Partnership 12. As temáticas abordadas contemplam: a qualidade do tempo dos 
pais com as crianças e adolescentes durante a pandemia de COVID-19; criação de espaços para falarem de seus sentimentos e angústias; uso de disciplina positiva como forma de lidar com comportamentos de desobediência; dicas de organização da rotina familiar, manutenção da calma e redução do estresse neste período; e dicas para manter o pensamento positivo durante o distanciamento social 12. A ONU Mulheres também elaborou um documento sobre os impactos e implicações da pandemia e as diferenças entre os gêneros 10 . Nessa publicação, reforça-se que em contexto de emergência há o aumento de violência contra a mulher por causa da dificuldade de rompimento das relações abusivas.

É preciso aproveitar as experiências já existentes e reforçar o que já vem sendo realizado por instituições governamentais e não governamentais em nosso país, adaptando estas iniciativas à situação específica que estamos vivendo no cenário da COVID-19. Nessa perspectiva, entendemos ser fundamental os seguintes pontos:

(1) Garantir o atendimento 24 horas do Ligue 180, Disque 100 (violação aos direitos humanos) e 190 (Polícia Civil), e a manutenção do trabalho dos Conselhos Tutelares por plantão presencial ou via telefone, WhatsApp, aplicativos para celulares e por meio digital para as denúncias de violação de direitos; (2) Garantir a agilidade do julgamento das denúncias de violência contra a mulher, que podem ser solicitadas pela vítima ao delegado(a) de polícia ou por meio do Ministério Público, visando à instalação de medidas protetivas de urgência, quando necessárias;

(3) Reforçar as campanhas publicitárias que tenham como foco central a importância de que todos "metam a colher em briga de marido e mulher". Da mesma forma, são necessárias as campanhas de alerta sobre os diferentes tipos de maus-tratos contra crianças e adolescentes. Vizinhos, parentes e amigos podem fazer toda a diferença em uma situação como essa;

(4) Incentivar as iniciativas de apoio às mulheres, crianças e adolescentes em situação de violência, baseando-se no acolhimento e aconselhamento psicológico, socioassistencial, jurídico e de saúde;

(5) Dentro do possível, é importante que mulheres em situação de violência busquem fazer o distanciamento social acompanhadas de outros familiares que não apenas o marido agressor e os filhos;

(6) Em situações extremas, é importante manter o telefone celular protegido, bem como telefones de familiares e amigos com quem as mulheres possam contar em situação de emergência, além de um plano de fuga seguro para a mulher e seus filhos.

Esperamos que este artigo tenha dado maior visibilidade ao problema, e tenha sensibilizado os governantes e a sociedade como um todo a incorporar essas estratégias no conjunto de intervenções que visem ao controle e à redução de danos da pandemia.

\section{Colaboradores}

E. S. Marques, C. L. Moraes e M. H. Hasselmann contribuíram com a concepção, redação e revisão crítica final do artigo. S. F. Deslandes e M. E. Reichenheim contribuíram com a redação e revisão crítica final do artigo.

\section{Informações adicionais}

ORCID: Emanuele Souza Marques (0000-00028633-7290); Claudia Leite de Moraes (0000-00023223-1634); Maria Helena Hasselmann (00000002-3106-1522); Suely Ferreira Deslandes (00000002-7062-3604); Michael Eduardo Reichenheim (0000-0001-7232-6745).

\section{Referências}

1. Guo YR, Cao QD, Hong ZS, Tan YY, Chen SD, Jin HJ, et al. The origin, transmission and clinical therapies on coronavirus disease 2019 (COVID-19) outbreak - an update on the status. Mil Med Res 2020; 7:11.

2. Johns Hopkins University. COVID-19 Global Cases by the Center for Systems Science and Engineering (CSSE). https://coronavirus.jhu. edu/map.html (acessado em 08/Abr/2020).

3. Ministério da Saúde. COVID-19: Painel Coronavírus. https://covid.saude.gov.br/ (acessado em 08/Abr/2020).

4. World Health Organization. WHO DirectorGeneral's opening remarks at the media briefing on COVID-19 - 16 March 2020. https:// www.who.int/dg/speeches/detail/who-direc tor-general-s-opening-remarks-at-the-mediabriefing-on-covid-19---16-march-2020 (acessado em 16/Mar/2020). 
5. Hellewell J, Abbott S, Gimma A, Bosse NI, Jarvis CI, Russell TW, et al. Feasibility of controlling COVID-19 outbreaks by isolation of cases and contacts. Lancet Glob Health 2020; 8:e488e96.

6. Adams V. Disasters and capitalism....and COVID-19. Somatosphere 2020. http://soma tosphere.net/2020/disaster-capitalism-covid 19.html/ (acessado em 26/Mar/2020).

7. Sullivan R, Chalkidou K. Urgent call for an exit plan: the economic and social consequences of responses to COVID-19 pandemic. Center for Global Development 2020. https://www.cgdev. org/blog/urgent-call-exit-plan-economic-andsocial-consequences-responses-covid-19-pan demic (acessado em 31/Mar/2020).

8. Ministério da Economia. Confira as medidas tomadas pelo Ministério da Economia em função da Covid-19 (Coronavírus). https:// www.gov.br/economia/pt-br/assuntos/noti cias $/ 2020 / \mathrm{marco} /$ confira-as-medidas-toma das-pelo-ministerio-da-economia-em-funcaodo-covid-19-coronavirus (acessado em 06/ Abr/2020).

9. End Violence Against Children. Protecting children during the COVID-19 outbreak: resources to reduce violence and abuse. https:// www.end-violence.org/protecting-childrenduring-covid-19-outbreak (acessado em 26/ Mar/2020)

10. ONU Mulheres Brasil. Gênero e COVID-19 na América Latina e no Caribe: dimensões de gênero na resposta. Brasília: ONU Mulheres Brasil; 2020.

11. Nações Unidas Brasil. Relatora da ONU: Estados devem combater violência doméstica na quarentena por COVID-19. https://nacoesuni das.org/relatora-da-onu-estados-devem-com bater-violencia-domestica-na-quarentena-porcovid-19/ (acessado em 27/Mar/2020).

12. World Health Organization. Coronavirus disease (COVID-19) advice for the public: healthy parenting. https://www.who.int/emergencies/ diseases/novel-coronavirus-2019/advice-forpublic/healthy-parenting (acessado em 30/ mar/2020).

13. World Health Organization; United Nations Children's Fund; End Violence Against Children; Internet of Good Things; Parenting for Longlife Health; United States Agency for International Development; et al. COVID-19 parenting. https://www.covid19parenting.com/ (acessado em 26/Mar/2020).

14. Cluver L, Lachman JM, Sherr L, Wessels I, Krug E, Rakotomalala S, et al. Parenting in a time of COVID-19. Lancet 2020; 395:e64.

15. Colbourn T. COVID-19: extending or relaxing distancing control measures. Lancet Public Health 2020; [Epub ahead of print].

16. Emanuel EJ, Persad G, Upshur R, Thome B, Parker M, Glickman A, et al. Fair allocation of scarce medical resources in the time of Covid-19. N Engl J Med 2020; [Epub ahead of print].
17. BBC News. Coronavirus: 'Domestic abuse pandemic likely due to shutdown'. https://www. bbc.com/news/uk-wales-52076789 (acessado em 30/Mar/2020).

18. BBC News. Coronavirus: la preocupación por las víctimas de violencia de género que tienen que convivir en cuarentena con su agresor (y dónde buscar ayuda). https://www.bbc.com/ mundo/noticias-52009140 (acessado em 24/ Mar/2020).

19. Ribeiro D. Com isolamento, a questão da violência contra a mulher fica ainda mais grave. Folha S.Paulo 2020; 27 mar. https://www1. folha.uol.com.br/colunas/djamila-ribei ro/2020/03/com-isolamento-a-questao-daviolencia-contra-a-mulher-fica-ainda-maisgrave.shtml.

20. Salisbury H. What might we learn from the covid-19 pandemic? BMJ 2020; 368:m1087.

21. Selvaratnam T. Where can domestic violence victims turn during Covid-19? The New York Times 2020; 23 mar. https://www.nytimes. com/2020/03/23/opinion/covid-domesticviolence.html.

22. Bassan P. Casos de violência doméstica no RJ crescem 50\% durante confinamento. G1 2020; 23 mar. https://g1.globo.com/rj/rio-de-janei ro/noticia/2020/03/23/casos-de-violenciadomestica-no-rj-crescem-50percent-duran te-confinamento.ghtml.

23. Godin M. As cities around the world go on lockdown, victims of domestic violence look for a way out. Time 2020; 18 mar. https://time. com/5803887/coronavirus-domestic-violencevictims/.

24. Golfieri M, Andrian A. O aumento da violência doméstica em tempos de Covid-19. Estadão 2020; 1 abr. https://politica.estadao.com.br/ blogs/fausto-macedo/o-aumento-da-violenciadomestica-em-tempos-de-covid-19/.

25. Women's Aid. The impact of COVID-19 on women and children experiencing domestic abuse, and the life-saving services that support them. https://www.womensaid.org.uk/theimpact-of-covid-19-on-women-and-childrenexperiencing-domesticabuse-and-the-life-sav ing-services-that-support-them/ (acessado em 17/Mar/2020).

26. World Health Organization. COVID-19 and violence against women: what the health sector/ system can do. Geneva: World Health Organization; 2020.

27. EURACTIV. Domestic violence increases in France during COVID-19 lockdown. https:// www.euractiv.com/section/politics/news/ domestic-violence-increases-in-france-dur ing-covid-19-lockdown/ (acessado em 30/ Mar/2020).

28. Galvani G. Violência doméstica na quarentena: como se proteger de um abusador? CartaCapital 2020; 29 mar. https://www.cartacapital. com.br/saude/violencia-domestica-na-quaren tena-como-se-proteger-de-um-abusador/. 
29. Barone I. Coronavírus: denúncias de violência doméstica aumentam e expõem impacto social da quarentena. Gazeta do Povo 2020; 28 mar. https://www.gazetadopovo.com.br/vida-e-ci dadania/coronavirus-denuncias-de-violenciadomestica-aumentam-e-expoem-impacto-so cial-da-quarentena/.

30. Leite C. Países registram aumento de violência doméstica durante período de quarentena; veja como denunciar casos no Ceará. O Povo Online 2020; 30 mar. https://www.opovo.com. $\mathrm{br} /$ coronavirus/2020/03/30/paises-registramaumento-de-violencia-domestica-durante-pe riodo-de-quarentena--veja-como-denunci ar-casos-no-ceara.html.

31. Moraes K. Quarentena do coronavírus eleva denúncias de violência doméstica no Brasil; saiba como se proteger. JC 2020; 30 mar. https:// jc.ne10.uol.com.br/brasil/2020/03/5604162quarentena-do-coronavirus--eleva-de nuncias-de-violencia-domestica-no-brasil-saiba-como-se-proteger.html.

32. Chan KL. Children exposed to child maltreatment and intimate partner violence: a study of co-occurrence among Hong Kong Chinese families. Child Abuse Negl 2011; 35:532-42.

33. Hamby S, Finkelhor D, Turner H, Ormrod R. The overlap of witnessing partner violence with child maltreatment and other victimizations in a nationally representative survey of youth. Child Abuse Negl 2010; 34:734-41.
34. Ko Ling C. Co-occurrence of intimate partner violence and child abuse in Hong Kong Chinese families. J Interpers Violence 2011; 26:1322-42.

35. Reichenheim ME, Dias AS, Moraes CL. Co-ocorrência de violência física conjugal e contra filhos em serviços de saúde. Rev Saúde Pública 2006; 40:595-603.

36. Taylor CA, Lee SJ, Guterman NB, Rice JC. Use of spanking for 3-year-old children and associated intimate partner aggression or violence. Pediatrics 2010; 126:415-24

37. Krug EG, Dahlberg LL, Mercy JA, Zwi AB, Lozano R. World report on violence and health. Geneva: World Health Organization; 2002.

38. United Nations Educational, Scientific and Cultural Organization. Covid-19 educational disruption and response. https://en.unesco. org/themes/education-emergencies/coro navirus-school-closures (acessado em 30/ Mar/2020).

39. World Health Organization. Joint Leaders' statement - violence against children: a hidden crisis of the COVID-19 pandemic. Geneva: World Health Organization; 2020.

40. The Alliance for Child Protection in Humanitarian Action. Technical note: protection of children during the coronavirus pandemic. v. 1. https://alliancecpha.org/en/COVD19. 